\title{
Intuitionistic Fuzzy Normal subrings over a non-associative ring
}

\author{
Tariq Shah, Nasreen Kausar and Inayatur-Rehman
}

\begin{abstract}
N. Palaniappan et. al $[20,28]$ have investigated the concept of intuitionistic fuzzy normal subrings in associative rings. In this study we extend these notions for a class of non-associative rings.
\end{abstract}

\section{Introduction}

In 1972, left almost semigroups (LA-semigroups) have been introduced by Kazim and Naseerudin [14]. A groupoid $S$ is called an LA-semigroup if it satisfies the left invertive law: $(a b) c=(c b) a$ for all $a, b, c \in S$. This structure is also known as Abel-Grassmann's groupoid (abbreviated as AG-groupoid) [21, 22]. Holgate [11], has called the same structure as left invertive groupoid. An AG-groupoid is the midway structure between a commutative semigroup and a groupoid. Actually an LA-semigroup is non-commutative and non-associative structure. In [13], a groupoid $S$ is called medial if $(a b)(c d)=(a c)(b d)$ for all $a, b, c, d \in S$, and $S$ is paramedial if $(a b)(c d)=(d b)(c a)$ (see [5, line 37]). Naturally every AG-groupoid satisfies medial law. In general an AG-groupoid needs not to be a paramedial. However, by [21] every AG-groupoid with left identity is paramedial. Ideals in AG-groupoids have been discussed in [21, 22].

In [15], Kamran extended the notion of LA-semigroup to left almost group (LA-group). A groupoid $G$ is called a left almost group (LA-group), if there

Key Words: (Intuitionistic) fuzzy set, (intuitionistic) fuzzy LA-subrings, (intuitionistic) fuzzy normal LA-subrings.

2010 Mathematics Subject Classification: 03F55, 08A72, 20N25

Received: May, 2011.

Revised: June, 2011.

Accepted: February, 2012. 
exists left identity $e \in G$ (that is $e a=a$ for all $a \in G$ ), for $a \in G$ there exists $b \in G$ such that $b a=e$ and left invertive law holds in $G$.

Shah and Rehman [25], have discussed left almost ring (LA-ring) of finitely nonzero functions which is in fact a generalization of a commutative semigroup ring. By a left almost ring, we mean a non-empty set $R$ with at least two elements such that $(R,+)$ is an LA-group, $(R, \cdot)$ is an LA-semigroup, both left and right distributive laws hold. For example, from a commutative ring $(R,+, \cdot)$, we can always obtain an LA-ring $(R, \oplus, \cdot)$ by defining for all $a, b \in R$, $a \oplus b=b-a$ and $a \cdot b$ is same as in the ring. An LA-ring is in fact a class of non-associative and non-commutative rings. Recently Shah and Rehman [26], investigated some properties of LA-rings through their ideals and intuitively ideal thoery would be a gate way for fuzzy concepts in LA-rings.

After the introduction of fuzzy set by Zadeh [31], several researchers explored on the generalization of the notion of fuzzy set. The concept of intuitionistic fuzzy set was introduced by Atanassov [1, 2], as a generalization of the notion of fuzzy set.

Fuzzy rings and fuzzy ideals have been discussed in [9]. In [8], Dib and Youssef have examined the properties of fuzzy cartesian product, fuzzy relations and fuzzy functions. Volf, has investigated the properties of fuzzy subfield in [29].

Intuitionistic fuzzy subrings and intuitionistic fuzzy ideals of a ring have been defined in [3, 4]. Palaniappan et. al [20] explored the notion of homomorphism, antihomomorphism of intuitionistic fuzzy normal subrings. Moreover intuitionistic fuzzy ring and its homomorphism image have been investigated by Yan [30]. Recently, some properties of intuitionistic fuzzy normal subrings have been discussed in [28].

In this study we followed lines as adopted in [20, 28] and established the notion of intuitionistic fuzzy normal LA-subrings of LA-rings. Specifically we show that: (1) An IFS $A=\left(\mu_{A}, \gamma_{A}\right)$ is an intuitionistic fuzzy normal LAsubring of an LA-ring $R$ if and only if the fuzzy sets $\mu_{A}$ and $\overline{\gamma_{A}}$ are fuzzy normal LA-subrings of $R$. (2) An IFS $A=\left(\mu_{A}, \gamma_{A}\right)$ is an intuitionistic fuzzy normal LA-subring of an LA-ring $R$ if and only if the fuzzy sets $\overline{\mu_{A}}$ and $\gamma_{A}$ are anti-fuzzy normal LA-subrings of $R$.

\section{Preliminaries}

Let $R$ be an LA-ring. By an LA-subring of $R$ we mean a non-empty subset $A$ of $R$ such that $a-b$ and $a b \in A$, for all $a, b \in A$, and by a left (right) ideal of $R$ we mean an LA-subring $A$ of $R$ such that $R A \subseteq A(A R \subseteq A)$, respectively. By two-sided ideal or simply ideal, we mean a non-empty subset $A$ of $R$ which is both a left and a right ideal of $R$. 
In the following we are giving an example of a finite LA-ring from [27].

Example 2.1. [27, Example 1] An LA-ring of order 5 :

\begin{tabular}{l|lllll}
+ & 0 & 1 & 2 & 3 & 4 \\
\hline 0 & 0 & 1 & 2 & 3 & 4 \\
1 & 4 & 0 & 1 & 2 & 3 \\
2 & 3 & 4 & 0 & 1 & 2 \\
3 & 2 & 3 & 4 & 0 & 1 \\
4 & 1 & 2 & 3 & 4 & 0
\end{tabular}

\begin{tabular}{cc|ccccc}
\multirow{4}{*}{ and } & $\cdot$ & 0 & 1 & 2 & 3 & 4 \\
\cline { 2 - 6 } & 0 & 0 & 0 & 0 & 0 & 0 \\
& 1 & 0 & 1 & 2 & 3 & 4 \\
& 2 & 0 & 2 & 4 & 1 & 3 \\
& 3 & 0 & 3 & 1 & 4 & 2 \\
& 4 & 0 & 4 & 3 & 2 & 1
\end{tabular}

As in [31], by a fuzzy set $\mu$ in a non-empty set $X$ we mean a function $\mu: X \rightarrow[0,1]$ and we denote the complement of $\mu$ by $\bar{\mu}(x)=1-\mu(x)$ for all $x \in X$.

An intuitionistic fuzzy set (briefly, IFS) $A$ in a non-empty set $X$ is an object having the form $A=\left\{\left(x, \mu_{A}(x), \gamma_{A}(x)\right): x \in X\right\}$, where the functions $\mu_{A}: X \rightarrow[0,1]$ and $\gamma_{A}: X \rightarrow[0,1]$ denote the degree of membership and the degree of nonmembership respectively, and $0 \leq \mu_{A}(x)+\gamma_{A}(x) \leq 1$ for all $x \in X$ (see $[1,2])$.

An intuitionistic fuzzy set $A=\left\{\left(x, \mu_{A}(x), \gamma_{A}(x)\right): x \in X\right\}$ in $X$ can be identified to an ordered pair $\left(\mu_{A}, \gamma_{A}\right)$ in $I^{X} \times I^{X}$, where $I^{X}$ is the set of all functions from $X$ to $[0,1]$. For the sake of simplicity, we shall use the symbol $A=\left(\mu_{A}, \gamma_{A}\right)$ for the IFS $A=\left\{\left(x, \mu_{A}(x), \gamma_{A}(x)\right): x \in X\right\}$.

\section{Intuitionistic fuzzy normal LA-subrings}

A fuzzy subset $\mu$ of an LA-ring $R$ is called a fuzzy LA-subring of $R$ if $\mu(x-y) \geq$ $\min \left\{\mu_{A}(x), \mu_{A}(y)\right\}$ and $\mu(x y) \geq \min \left\{\mu_{A}(x), \mu_{A}(y)\right\}$ for all $x, y \in R$ [23]. A fuzzy subset $\mu$ of an LA-ring $R$ is called an anti fuzzy LA-subring of $R$ if $\mu(x-y) \leq \max \left\{\mu_{A}(x), \mu_{A}(y)\right\}$ and $\mu(x y) \leq \max \left\{\mu_{A}(x), \mu_{A}(y)\right\}$ for all $x, y \in R$. A fuzzy LA-subring of an LA-ring $R$ is called a fuzzy normal LAsubring of $R$ if $\mu(x y)=\mu(y x)$ for all $x, y \in R$. Similarly for anti-fuzzy normal LA-subring.

Definition 3.1. [24] An IFS $A=\left(\mu_{A}, \gamma_{A}\right)$ in $R$ is called an intuitionistic fuzzy LA-subring IFLS(IFLSR) of $R$ if

(a) $\mu_{A}(x-y) \geq \min \left\{\mu_{A}(x), \mu_{A}(y)\right\}$,

(b) $\gamma_{A}(x-y) \leq \max \left\{\gamma_{A}(x), \gamma_{A}(y)\right\}$,

(c) $\mu_{A}(x y) \geq \min \left\{\mu_{A}(x), \mu_{A}(y)\right\}$,

(d) $\gamma_{A}(x y) \leq \max \left\{\gamma_{A}(x), \gamma_{A}(y)\right\}$,

for all $x, y \in R$.

Intuitionistic fuzzy LA-subring is an extension of fuzzy LA-subring. 
Let $R$ be an LA-ring. An intuitionistic fuzzy LA-subring $A=\left(\mu_{A}, \gamma_{A}\right)$ of $R$ is said to be an intuitionistic fuzzy normal LA-subring (IFNLSR) of $R$ if $\mu_{A}(x y)=\mu_{A}(y x)$ and $\gamma_{A}(x y)=\gamma_{A}(y x)$ for all $x, y \in R$.

Example 3.2. Let $R=\{0,1,2,3\}$. Define + and $\cdot$ in $R$ as follows :

$\begin{array}{lllll}+ & 0 & 1 & 2 & 3 \\ 0 & 0 & 1 & 2 & 3 \\ 1 & 3 & 0 & 1 & 2 \\ 2 & 2 & 3 & 0 & 1 \\ 3 & 1 & 2 & 3 & 0\end{array}$

\begin{tabular}{cccccc} 
& & 0 & 1 & 2 & 3 \\
\multirow{3}{*}{ and } & 0 & 0 & 0 & 0 & 0 \\
& 1 & 0 & 1 & 2 & 3 \\
& 2 & 0 & 2 & 0 & 2 \\
& 3 & 0 & 3 & 2 & 1
\end{tabular}

Then $R$ is an LA-ring. Let an IFS $A=\left(\mu_{A}, \gamma_{A}\right)$ of $R$. We define $\mu_{A}(0)=$ $\mu_{A}(2)=0.7, \mu_{A}(1)=\mu_{A}(3)=0$ and $\gamma_{A}(0)=\gamma_{A}(2)=0, \gamma_{A}(1)=\gamma_{A}(3)=0.7$. Then $A=\left(\mu_{A}, \gamma_{A}\right)$ is an intuitionistic fuzzy normal LA-subring of $R$.

Remark 3.3. $S=\{0,2\}$ is an LA-subring of $R$.

Definition 3.4. [24] Let $R$ be an LA-ring and $A$ be a non-empty subset of $R$. The intuitionistic characteristic function of $A$ is denoted by $\chi_{A}=$ $\left\langle\mu_{\chi_{A}}, \gamma_{\chi_{A}}\right\rangle$ and is defined by

$$
\mu_{\chi_{A}}: R \rightarrow[0,1] \mid x \rightarrow \mu_{\chi_{A}}(x):=\left\{\begin{array}{l}
1 \text { if } x \in A \\
0 \text { if } x \notin A
\end{array}\right.
$$

and

$$
\gamma_{\chi_{A}}: R \rightarrow[0,1] \mid x \rightarrow \gamma_{\chi_{A}}(x):=\left\{\begin{array}{l}
0 \text { if } x \in A \\
1 \text { if } x \notin A
\end{array}\right.
$$

Lemma 3.5. If $A$ is a subset of an LA-ring $R$, then $A$ is an LA-subring of $R$ if and only if the intuitionistic characteristic function $\chi_{A}=\left\langle\mu_{\chi_{A}}, \gamma_{\chi_{A}}\right\rangle$ of $A$ is an intuitionistic fuzzy normal LA-subring of $R$.

Proof. Let $A$ be an LA-subring of $R$ and $a, b \in R$. If $a, b \in A$, then by definition of characteristic function $\mu_{\chi_{A}}(a)=1=\mu_{\chi_{A}}(b)$ and $\gamma_{\chi_{A}}(a)=0=\gamma_{\chi_{A}}(b)$. $A$ being an LA-subring, $a-b$ and $a b \in A$. It follows that $\mu_{\chi_{A}}(a-b)=1=1 \wedge 1=$ $\mu_{\chi_{A}}(a) \wedge \mu_{\chi_{A}}(b)$ and $\mu_{\chi_{A}}(a b)=1=1 \wedge 1=\mu_{\chi_{A}}(a) \wedge \mu_{\chi_{A}}(b)$. This imply that $\mu_{\chi_{A}}(a-b) \geq \min \left\{\mu_{\chi_{A}}(a), \mu_{\chi_{A}}(b)\right\}$ and $\mu_{\chi_{A}}(a b) \geq \min \left\{\mu_{\chi_{A}}(a), \mu_{\chi_{A}}(b)\right\}$. Now $\gamma_{\chi_{A}}(a-b)=0=0 \vee 0=\gamma_{\chi_{A}}(a) \vee \gamma_{\chi_{A}}(b)$ and $\gamma_{\chi_{A}}(a b)=0=0 \vee$ $0=\gamma_{\chi_{A}}(a) \vee \gamma_{\chi_{A}}(b)$. This imply that $\gamma_{\chi_{A}}(a-b) \leq \max \left\{\gamma_{\chi_{A}}(a), \gamma_{\chi_{A}}(b)\right\}$ and $\gamma_{\chi_{A}}(a b) \leq \max \left\{\gamma_{\chi_{A}}(a), \gamma_{\chi_{A}}(b)\right\}$. Since $a b$ and $b a \in A$, it follows that $\mu_{\chi_{A}}(a b)=1=\mu_{\chi_{A}}(b a)$ and $\gamma_{\chi_{A}}(a b)=0=\gamma_{\chi_{A}}(b a)$. Consequently $\mu_{\chi_{A}}(a b)=$ $\mu_{\chi_{A}}(b a)$ and $\gamma_{\chi_{A}}(a b)=\gamma_{\chi_{A}}(b a)$. Similarly we can prove that

$$
\begin{aligned}
\mu_{\chi_{A}}(a-b) & \geq \min \left\{\mu_{\chi_{A}}(a), \mu_{\chi_{A}}(b)\right\}, \mu_{\chi_{A}}(a b) \geq \min \left\{\mu_{\chi_{A}}(a), \mu_{\chi_{A}}(b)\right\} \\
\gamma_{\chi_{A}}(a-b) & \leq \max \left\{\gamma_{\chi_{A}}(a), \gamma_{\chi_{A}}(b)\right\}, \gamma_{\chi_{A}}(a b) \leq \max \left\{\gamma_{\chi_{A}}(a), \gamma_{\chi_{A}}(b)\right\}, \\
\mu_{\chi_{A}}(a b) & =\mu_{\chi_{A}}(b a) \text { and } \gamma_{\chi_{A}}(a b)=\gamma_{\chi_{A}}(b a),
\end{aligned}
$$


when $a, b \notin A$. Thus the intuitionistic characteristic function $\chi_{A}=\left\langle\mu_{\chi_{A}}, \gamma_{\chi_{A}}\right\rangle$ of $A$ is an intuitionistic fuzzy normal LA-subring of $R$.

Conversely, assume that the intuitionistic characteristic function $\chi_{A}=$ $\left\langle\mu_{\chi_{A}}, \gamma_{\chi_{A}}\right\rangle$ of $A$ is an intuitionistic fuzzy normal LA-subring of $R$. Let $a, b \in A$. By definition $\mu_{\chi_{A}}(a)=1=\mu_{\chi_{A}}(b)$ and $\gamma_{\chi_{A}}(a)=0=\gamma_{\chi_{A}}(b)$. By hypothesis

$$
\begin{aligned}
\mu_{\chi_{A}}(a-b) & \geq \mu_{\chi_{A}}(a) \wedge \mu_{\chi_{A}}(b)=1 \wedge 1=1, \\
\mu_{\chi_{A}}(a b) & \geq \mu_{\chi_{A}}(a) \wedge \mu_{\chi_{A}}(b)=1 \wedge 1=1, \\
\gamma_{\chi_{A}}(a-b) & \leq \gamma_{\chi_{A}}(a) \vee \gamma_{\chi_{A}}(b)=0 \vee 0=0, \\
\gamma_{\chi_{A}}(a b) & \leq \gamma_{\chi_{A}}(a) \vee \gamma_{\chi_{A}}(b)=0 \vee 0=0,
\end{aligned}
$$

This imply that $\mu_{\chi_{A}}(a-b)=1, \mu_{\chi_{A}}(a b)=1$ and $\gamma_{\chi_{A}}(a-b)=0, \gamma_{\chi_{A}}(a b)=$ 0 . Thus $a-b$ and $a b \in A$. Hence $A$ is an LA-subring of $R$.

If $A$ and $B$ are two LA-subrings of an LA-ring $R$, then their intersection $A \cap B$ is also an LA-subring of $R$.

Lemma 3.6. If $A$ and $B$ are two LA-subrings of an LA-ring $R$, then their intersection $A \cap B$ is an LA-subring of $R$ if and only if the intuitionistic characteristic function $\chi_{C}=\left\langle\mu_{\chi_{c}}, \gamma_{\chi_{c}}\right\rangle$ of $C=A \cap B$ is an intuitionistic fuzzy normal LA-subring of $R$.

Proof. Let $C=A \cap B$ be an LA-subring of $R$ and $a, b \in R$. If $a, b \in C=$ $A \cap B$, then by definition of characteristic function $\mu_{\chi_{C}}(a)=1=\mu_{\chi_{C}}(b)$ and $\gamma_{\chi_{C}}(a)=0=\gamma_{\chi_{C}}(b)$. Since $a-b, a b \in A$ and $B$, it follows that $a-b$ and $a b \in C$. Thus $\mu_{\chi_{C}}(a-b)=1=1 \wedge 1=\mu_{\chi_{C}}(a) \wedge \mu_{\chi_{C}}(b)$ and $\mu_{\chi_{C}}(a b)=$ $1=1 \wedge 1=\mu_{\chi_{C}}(a) \wedge \mu_{\chi_{C}}(b)$. Thus $\mu_{\chi_{C}}(a-b) \geq \min \left\{\mu_{\chi_{C}}(a), \mu_{\chi_{C}}(b)\right\}$ and $\mu_{\chi_{C}}(a b) \geq \min \left\{\mu_{\chi_{C}}(a), \mu_{\chi_{C}}(b)\right\}$. Now $\gamma_{\chi_{C}}(a-b)=0=0 \vee 0=\gamma_{\chi_{C}}(a) \vee$ $\gamma_{\chi_{C}}(b)$ and $\gamma_{\chi_{C}}(a b)=0=0 \vee 0=\gamma_{\chi_{C}}(a) \vee \gamma_{\chi_{C}}(b)$. Thus $\gamma_{\chi_{C}}(a-b) \leq$ $\max \left\{\gamma_{\chi_{C}}(a), \gamma_{\chi_{C}}(b)\right\}$ and $\gamma_{\chi_{C}}(a b) \leq \max \left\{\gamma_{\chi_{C}}(a), \gamma_{\chi_{C}}(b)\right\}$. As $a b$ and $b a \in$ $C$, so $\mu_{\chi_{C}}(a b)=1=\mu_{\chi_{C}}(b a)$ and $\gamma_{\chi_{C}}(a b)=0=\gamma_{\chi_{C}}(b a)$. Accordingly $\mu_{\chi_{C}}(a b)=\mu_{\chi_{C}}(b a)$ and $\gamma_{\chi_{C}}(a b)=\gamma_{\chi_{C}}(b a)$. Similarly we have

$$
\begin{aligned}
\mu_{\chi_{C}}(a-b) & \geq \min \left\{\mu_{\chi_{C}}(a), \mu_{\chi_{C}}(b)\right\}, \mu_{\chi_{C}}(a b) \geq \min \left\{\mu_{\chi_{C}}(a), \mu_{\chi_{C}}(b)\right\} \\
\gamma_{\chi_{C}}(a-b) & \leq \max \left\{\gamma_{\chi_{C}}(a), \gamma_{\chi_{C}}(b)\right\}, \gamma_{\chi_{C}}(a b) \leq \max \left\{\gamma_{\chi_{C}}(a), \gamma_{\chi_{C}}(b)\right\} \\
\gamma_{\chi_{C}}(a b) & =\gamma_{\chi_{C}}(b a) \text { and } \gamma_{\chi_{C}}(a b)=\gamma_{\chi_{C}}(b a)
\end{aligned}
$$

when $a, b \notin C$. Hence the intuitionistic characteristic function $\chi_{C}=$ $\left\langle\mu_{\chi_{C}}, \gamma_{\chi_{C}}\right\rangle$ of $C$ is an intuitionistic fuzzy normal LA-subring of $R$.

Conversely, assume that the intuitionistic characteristic function $\chi_{C}=$ $\left\langle\mu_{\chi_{C}}, \gamma_{\chi_{C}}\right\rangle$ of $C=A \cap B$ is an intuitionistic fuzzy normal LA-subring of 
R. Let $a, b \in C=A \cap B$. This imply that $\mu_{\chi_{C}}(a)=1=\mu_{\chi_{C}}(b)$ and $\gamma_{\chi_{C}}(a)=0=\gamma_{\chi_{C}}(b)$. By our supposition

$$
\begin{aligned}
\mu_{\chi_{C}}(a-b) & \geq \mu_{\chi_{C}}(a) \wedge \mu_{\chi_{C}}(b)=1 \wedge 1=1, \\
\mu_{\chi_{C}}(a b) & \geq \mu_{\chi_{C}}(a) \wedge \mu_{\chi_{C}}(b)=1 \wedge 1=1, \\
\gamma_{\chi_{C}}(a-b) & \leq \gamma_{\chi_{C}}(a) \vee \gamma_{\chi_{C}}(b)=0 \vee 0=0, \\
\gamma_{\chi_{C}}(a b) & \leq \gamma_{\chi_{C}}(a) \vee \gamma_{\chi_{C}}(b)=0 \vee 0=0,
\end{aligned}
$$

This imply that $\mu_{\chi_{C}}(a-b)=1, \mu_{\chi_{C}}(a b)=1$ and $\gamma_{\chi_{C}}(a-b)=0, \gamma_{\chi_{C}}(a b)=$ 0 . Thus $a-b$ and $a b \in C$. Hence $C$ is an LA-subring of $R$.

Corollary 3.7. If $\left\{A_{i}\right\}_{i \in I}$ is a family of LA-subrings of $R$, then $C=\cap A_{i}$ is an LA-subring of $R$, where $\cap A_{i}=\left(\wedge \mu_{A_{i}}, \vee \gamma_{A_{i}}\right)$ and

$$
\begin{aligned}
& \wedge \mu_{A_{i}}(x)=\inf \left\{\mu_{A_{i}}(x): i \in I, x \in R\right\}, \\
& \vee \gamma_{A_{i}}(x)=\sup \left\{\gamma_{A_{i}}(x): i \in I, x \in R\right\},
\end{aligned}
$$

if and only if the intuitionistic characteristic function $\chi_{C}=\left\langle\mu_{\chi_{c}}, \gamma_{\chi_{c}}\right\rangle$ of $C=\cap A_{i}$ is an intuitionistic fuzzy normal LA-subring of $R$.

Theorem 3.8. If $A$ and $B$ are two intuitionistic fuzzy normal LA-subrings of an LA-ring $R$, then their intersection $A \cap B$ is an intuitionistic fuzzy normal LA-subring of $R$.

Proof. Let $A=\left\{\left(x, \mu_{A}(x), \gamma_{A}(x)\right): x \in R\right\}$ and $B=\left\{\left(x, \mu_{B}(x), \gamma_{B}(x)\right): x \in\right.$ $R\}$ be intuitionistic fuzzy normal LA-subrings of an LA-ring $R$. Let $C=A \cap B$ and $C=\left\{\left(x, \mu_{C}(x), \gamma_{C}(x)\right) \mid x \in R\right\}$, where $\mu_{C}(x)=\min \left\{\mu_{A}(x), \mu_{B}(x)\right\}$ and 


$$
\begin{aligned}
& \gamma_{C}(x)=\max \left\{\gamma_{A}(x), \gamma_{B}(x)\right\} . \text { Now } \\
& \mu_{C}(x-y)=\min \left\{\mu_{A}(x-y), \mu_{B}(x-y)\right\} \\
& =\mu_{A}(x-y) \wedge \mu_{B}(x-y) \\
& \geq\left\{\mu_{A}(x) \wedge \mu_{A}(y)\right\} \wedge\left\{\mu_{B}(x) \wedge \mu_{B}(y)\right\} \\
& =\mu_{A}(x) \wedge\left\{\mu_{A}(y) \wedge \mu_{B}(x)\right\} \wedge \mu_{B}(y) \\
& =\mu_{A}(x) \wedge\left\{\mu_{B}(x) \wedge \mu_{A}(y)\right\} \wedge \mu_{B}(y) \\
& =\left\{\mu_{A}(x) \wedge \mu_{B}(x)\right\} \wedge\left\{\mu_{A}(y) \wedge \mu_{B}(y)\right\} \\
& =\mu_{C}(x) \wedge \mu_{C}(y) \text {. } \\
& \mu_{C}(x y)=\min \left\{\mu_{A}(x y), \mu_{B}(x y)\right\} \\
& =\mu_{A}(x y) \wedge \mu_{B}(x y) \\
& \geq\left\{\mu_{A}(x) \wedge \mu_{A}(y)\right\} \wedge\left\{\mu_{B}(x) \wedge \mu_{B}(y)\right\} \\
& =\mu_{A}(x) \wedge\left\{\mu_{A}(y) \wedge \mu_{B}(x)\right\} \wedge \mu_{B}(y) \\
& =\mu_{A}(x) \wedge\left\{\mu_{B}(x) \wedge \mu_{A}(y)\right\} \wedge \mu_{B}(y) \\
& =\left\{\mu_{A}(x) \wedge \mu_{B}(x)\right\} \wedge\left\{\mu_{A}(y) \wedge \mu_{B}(y)\right\} \\
& =\mu_{C}(x) \wedge \mu_{C}(y) \text {. }
\end{aligned}
$$

Similarly $\gamma_{C}(x-y) \leq \gamma_{C}(x) \vee \gamma_{C}(y)$ and $\gamma_{C}(x y) \leq \gamma_{C}(x) \vee \gamma_{C}(y)$. Thus $C$ is an intuitionistic fuzzy LA-subring of an LA-ring $R$. Now $\mu_{C}(x y)=$ $\min \left\{\mu_{A}(x y), \mu_{B}(x y)\right\}=\min \left\{\mu_{A}(y x), \mu_{B}(y x)\right\}=\mu_{C}(y x)$. Similarly $\gamma_{C}(x y)=$ $\gamma_{C}(y x)$. Hence $A \cap B$ is an intuitionistic fuzzy normal LA-subring of $R$.

Proposition 3.9. If $A$ is an intuitionistic fuzzy normal LA-subring of an LA-ring $R$, then $\square A$ : $=\left(\mu_{A}, \overline{\mu_{A}}\right)$ is an intuitionistic fuzzy normal LA-subring of an LA-ring $R$.

Proof. We have to show that $\square A:=\left(\mu_{A}, \overline{\mu_{A}}\right)$ is an intuitionistic fuzzy normal LA-subring of $R$.

$$
\begin{aligned}
\overline{\mu_{A}}(x-y) & =1-\mu_{A}(x-y) \leq 1-\min \left\{\mu_{A}(x), \mu_{A}(y)\right\} \\
& =\max \left\{1-\mu_{A}(x), 1-\mu_{A}(y)\right\}=\max \left\{\overline{\mu_{A}}(x), \overline{\mu_{A}}(y)\right\} \\
& \Rightarrow \overline{\mu_{A}}(x-y) \leq \max \left\{\overline{\mu_{A}}(x), \overline{\mu_{A}}(y)\right\} . \\
\overline{\mu_{A}}(x y) & =1-\mu_{A}(x y) \leq 1-\min \left\{\mu_{A}(x), \mu_{A}(y)\right\} \\
& =\max \left\{1-\mu_{A}(x), 1-\mu_{A}(y)\right\}=\max \left\{\overline{\mu_{A}}(x), \overline{\mu_{A}}(y)\right\} \\
& \Rightarrow \overline{\mu_{A}}(x y) \leq \max \left\{\overline{\mu_{A}}(x), \overline{\mu_{A}}(y)\right\} . \\
\overline{\mu_{A}}(x y) & =1-\mu_{A}(x y)=1-\mu_{A}(y x)=\overline{\mu_{A}}(y x) .
\end{aligned}
$$


Proposition 3.10. If $A$ is an intuitionistic fuzzy normal LA-subring of an LA-ring $R$, then $\diamond A=\left(\overline{\gamma_{A}}, \gamma_{A}\right)$ is an intuitionistic fuzzy normal LA-subring of an LA-ring $R$.

Proof. We have to show that $\diamond A=\left(\overline{\gamma_{A}}, \gamma_{A}\right)$ is an intuitionistic fuzzy normal LA-subring of $R$.

$$
\begin{aligned}
\overline{\gamma_{A}}(x-y) & =1-\gamma_{A}(x-y) \geq 1-\max \left\{\gamma_{A}(x), \gamma_{A}(y)\right\} \\
& =\min \left\{1-\gamma_{A}(x), 1-\gamma_{A}(y)\right\}=\min \left\{\overline{\gamma_{A}}(x), \overline{\gamma_{A}}(y)\right\} \\
& \Rightarrow \overline{\gamma_{A}}(x-y) \geq \min \left\{\overline{\gamma_{A}}(x), \overline{\gamma_{A}}(y)\right\} . \\
\overline{\gamma_{A}}(x y) & =1-\gamma_{A}(x y) \geq 1-\max \left\{\gamma_{A}(x), \gamma_{A}(y)\right\} \\
& =\min \left\{1-\gamma_{A}(x), 1-\gamma_{A}(y)\right\}=\min \left\{\overline{\gamma_{A}}(x), \overline{\gamma_{A}}(y)\right\} \\
& \Rightarrow \overline{\gamma_{A}}(x y) \geq \min \left\{\overline{\gamma_{A}}(x), \overline{\gamma_{A}}(y)\right\} . \\
\overline{\gamma_{A}}(x y) & =1-\gamma_{A}(x y)=1-\gamma_{A}(y x)=\overline{\gamma_{A}}(y x) .
\end{aligned}
$$

Theorem 3.11. An IFS $A=\left(\mu_{A}, \gamma_{A}\right)$ is an intuitionistic fuzzy normal LA-subring of an LA-ring $R$ if and only if the fuzzy subsets $\mu_{A}$ and $\overline{\gamma_{A}}$ are fuzzy normal LA-subrings of $R$.

Proof. Let $A=\left(\mu_{A}, \gamma_{A}\right)$ be an intuitionistic fuzzy normal LA-subring of $R$. Then clearly $\mu_{A}$ is fuzzy normal LA-subring of $R$. Now

$$
\begin{aligned}
\overline{\gamma_{A}}(x-y) & =1-\gamma_{A}(x-y) \\
& \geq 1-\max \left\{\gamma_{A}(x), \gamma_{A}(y)\right\} \\
& =\min \left\{1-\gamma_{A}(x), 1-\gamma_{A}(y)\right\} \\
& =\min \left\{\overline{\gamma_{A}}(x), \overline{\gamma_{A}}(y)\right\} . \\
\overline{\gamma_{A}}(x y) & =1-\gamma_{A}(x y) \\
& \geq 1-\max \left\{\gamma_{A}(x), \gamma_{A}(y)\right\} \\
& =\min \left\{1-\gamma_{A}(x), 1-\gamma_{A}(y)\right\} \\
& =\min \left\{\overline{\gamma_{A}}(x), \overline{\gamma_{A}}(y)\right\} . \\
\overline{\gamma_{A}}(x y) & =1-\gamma_{A}(x y)=1-\gamma_{A}(y x)=\overline{\gamma_{A}}(y x) .
\end{aligned}
$$

Thus $\overline{\gamma_{A}}$ is a fuzzy normal LA-subring of $R$. 
Conversely, $\mu_{A}$ and $\overline{\gamma_{A}}$ are fuzzy normal LA-subrings of $R$.

$$
\begin{aligned}
1-\gamma_{A}(x-y) & =\overline{\gamma_{A}}(x-y) \geq \min \left\{\overline{\gamma_{A}}(x), \overline{\gamma_{A}}(y)\right\} \\
& =\min \left\{\left(1-\gamma_{A}(x)\right),\left(1-\gamma_{A}(y)\right)\right\} \\
& =1-\max \left\{\gamma_{A}(x), \gamma_{A}(y)\right\} \\
& \Rightarrow \gamma_{A}(x-y) \leq \max \left\{\gamma_{A}(x), \gamma_{A}(y)\right\} \\
1-\gamma_{A}(x y) & =\overline{\gamma_{A}}(x y) \geq \min \left\{\overline{\gamma_{A}}(x), \overline{\gamma_{A}}(y)\right\} \\
& =\min \left\{\left(1-\gamma_{A}(x)\right),\left(1-\gamma_{A}(y)\right)\right\} \\
& =1-\max \left\{\gamma_{A}(x), \gamma_{A}(y)\right\} \\
& \Rightarrow \gamma_{A}(x y) \leq \max \left\{\gamma_{A}(x), \gamma_{A}(y)\right\} . \\
1-\gamma_{A}(x y) & =\overline{\gamma_{A}}(x y)=\overline{\gamma_{A}}(y x)=1-\gamma_{A}(y x) \\
& \Rightarrow \gamma_{A}(x y)=\gamma_{A}(y x) .
\end{aligned}
$$

Thus $A=\left(\mu_{A}, \gamma_{A}\right)$ is an intuitionistic fuzzy normal LA-subring of an LAring $R$.

Theorem 3.12. An IFS $A=\left(\mu_{A}, \gamma_{A}\right)$ is an intuitionistic fuzzy normal LA-subring of an LA-ring $R$ if and only if the fuzzy subsets $\overline{\mu_{A}}$ and $\gamma_{A}$ are anti-fuzzy normal LA-subrings of $R$.

Proof. Suppose $A=\left(\mu_{A}, \gamma_{A}\right)$ is an intuitionistic fuzzy normal LA-subring of an LA-ring $R$. Clear $\gamma_{A}$ is an anti fuzzy normal LA-subring of $R$. Now we have to show that $\overline{\mu_{A}}$ is also an anti fuzzy normal LA-subring of $R$.

$$
\begin{aligned}
\overline{\mu_{A}}(x-y) & =1-\mu_{A}(x-y) \leq 1-\min \left\{\mu_{A}(x), \mu_{A}(y)\right\} \\
& =\max \left\{1-\mu_{A}(x), 1-\mu_{A}(y)\right\} \\
& =\max \left\{\overline{\mu_{A}}(x), \overline{\mu_{A}}(y)\right\} \\
& \Rightarrow \overline{\mu_{A}}(x-y) \leq \max \left\{\overline{\mu_{A}}(x), \overline{\mu_{A}}(y)\right\} . \\
\overline{\mu_{A}}(x y) & =1-\mu_{A}(x y) \leq 1-\min \left\{\mu_{A}(x), \mu_{A}(y)\right\} \\
& =\max \left\{1-\mu_{A}(x), 1-\mu_{A}(y)\right\} \\
& =\max \left\{\overline{\mu_{A}}(x), \overline{\mu_{A}}(y)\right\} \\
& \Rightarrow \overline{\mu_{A}}(x y) \leq \max \left\{\overline{\mu_{A}}(x), \overline{\mu_{A}}(y)\right\} . \\
\overline{\mu_{A}}(x y) & =1-\mu_{A}(x y)=1-\mu_{A}(y x)=\overline{\mu_{A}}(y x) .
\end{aligned}
$$

Hence $\overline{\mu_{A}}$ and $\gamma_{A}$ are anti fuzzy normal LA-subrings of $R$.

Conversely, suppose that $\overline{\mu_{A}}$ and $\gamma_{A}$ are anti fuzzy normal LA-subrings of $R$. Now we have to show that $A=\left(\mu_{A}, \gamma_{A}\right)$ is an intuitionistic fuzzy normal 
LA-subring of an LA-ring $R$.

$$
\begin{aligned}
1-\mu_{A}(x-y) & =\overline{\mu_{A}}(x-y) \leq \max \left\{\overline{\mu_{A}}(x), \overline{\mu_{A}}(y)\right\} \\
& =\max \left\{1-\mu_{A}(x), 1-\mu_{A}(y)\right\} \\
& =1-\min \left\{\mu_{A}(x), \mu_{A}(y)\right\} \\
& \Rightarrow \mu_{A}(x-y) \geq \min \left\{\mu_{A}(x), \mu_{A}(y)\right\} . \\
1-\mu_{A}(x y) & =\overline{\mu_{A}}(x y) \leq \max \left\{\overline{\mu_{A}}(x), \overline{\mu_{A}}(y)\right\} \\
& =\max \left\{1-\mu_{A}(x), 1-\mu_{A}(y)\right\} \\
& =1-\min \left\{\mu_{A}(x), \mu_{A}(y)\right\} \\
& \Rightarrow \mu_{A}(x y) \geq \min \left\{\mu_{A}(x), \mu_{A}(y)\right\} . \\
1-\mu_{A}(x y) & =\overline{\mu_{A}}(x y)=\overline{\mu_{A}}(y x)=1-\mu_{A}(y x) \\
& \Rightarrow \mu_{A}(x y)=\mu_{A}(y x) .
\end{aligned}
$$

Thus $A=\left(\mu_{A}, \gamma_{A}\right)$ is an intuitionistic fuzzy normal LA-subring of an LAring $R$.

\section{Direct Product of LA-rings}

In this section we discuss the direct product of LA-rings. Specifically, we show that if $A$ and $B$ are two LA-subrings of LA-rings $R_{1}$ and $R_{2}$ respectively, then $A \times B$ is an LA-subring of $R_{1} \times R_{2}$ if and only if the intuitionistic characteristic function $\chi_{C}=\left\langle\mu_{\chi_{C}}, \gamma_{\chi_{C}}\right\rangle$ of $C=A \times B$ is an intuitionistic fuzzy normal LAsubring of $R_{1} \times R_{2}$.

If $R_{1}, R_{2}$ are LA-rings, then direct product $R_{1} \times R_{2}$ of $R_{1}$ and $R_{2}$ is an LA-ring with pointwise addition ' + ' and multiplication ' $\circ$ ' defined as $(a, b)+$ $(c, d)=(a+c, b+d)$ and $(a, b) \circ(c, d)=(a c, b d)$, respectively for every $(a, b),(c, d)$ in $R_{1} \times R_{2}$. Likewise the direct product $R=\times_{i \in \Omega} R_{i}$ of a family of LA-rings $\left\{R_{i}: i \in \Omega\right\}$ has the structure of an LA-ring with the operations of addition and multiplication defined as

$$
\begin{aligned}
a+b & =\left(a_{1}, a_{2}, a_{3}, \ldots\right)+\left(b_{1}, b_{2}, b_{3}, \ldots\right) \\
& =\left(a_{1}+b_{1}, a_{2}+b_{2}, a_{3}+b_{3}, \ldots\right) \\
\text { and } a \circ b & =\left(a_{1}, a_{2}, a_{3}, \ldots\right) \circ\left(b_{1}, b_{2}, b_{3}, \ldots\right) \\
& =\left(a_{1} b_{1}, a_{2} b_{2}, a_{3} b_{3}, \ldots\right)
\end{aligned}
$$

for all $a, b \in R$.

Example 4.1. Let $R_{1}=\{0,1,2,3,4\}$ and $R_{2}=\{0,1,2,3\}$. Define addition and multiplication in $R_{1}$ and $R_{2}$ as in Example 2 and Example 3, respectively. Then $R_{1} \times R_{2}$ is an LA-ring with pointwise addition ' + ' and multipli- 
cation ' $\circ$ ' defined as $(a, b)+(c, d)=(a+c, b+d)$ and $(a, b) \circ(c, d)=(a c, b d)$, respectively for every $(a, b),(c, d)$ in $R_{1} \times R_{2}$.

$A=R_{1}=\{0,1,2,3,4\}$ and $B=\{0,2\}$ being LA-subrings of $R_{1}$ and $R_{2}$, then $A \times B$ is an LA-subring of $R_{1} \times R_{2}$ under the same operations defined as in $R_{1} \times R_{2}$.

Lemma 4.2. If $A$ and $B$ are two LA-subrings of LA-rings $R_{1}$ and $R_{2}$ respectively, then $A \times B$ is also an LA-subring of $R_{1} \times R_{2}$ under the same operations defined as in $R_{1} \times R_{2}$.

Proof. Straight forward.

Let $A$ and $B$ be two intuitionistic fuzzy subsets of LA-rings $R_{1}$ and $R_{2}$, respectively. The direct product of $A$ and $B$, is denoted by $A \times B$, is defined as $A \times B=\left\{\left((x, y), \mu_{A \times B}((x, y)), \gamma_{A \times B}((x, y))\right) \mid\right.$ for all $x \in R_{1}$ and $\left.y \in R_{2}\right\}$, where $\mu_{A \times B}((x, y))=\min \left\{\mu_{A}(x), \mu_{B}(y)\right\}$ and $\gamma_{A \times B}((x, y))=$ $\max \left\{\gamma_{A}(x), \gamma_{B}(y)\right\}$.

Theorem 4.3. Let $A$ and $B$ be two LA-subrings of LA-rings $R_{1}$ and $R_{2}$, respectively. The $A \times B$ is an LA-subring of $R_{1} \times R_{2}$ if and only if the intuitionistic characteristic function $\chi_{C}=\left\langle\mu_{\chi_{C}}, \gamma_{\chi_{C}}\right\rangle$ of $C=A \times B$ is an intuitionistic fuzzy normal LA-subring of $R_{1} \times R_{2}$.

Proof. Let $C=A \times B$ be an LA-subring of $R_{1} \times R_{2}$ and $a, b \in R_{1} \times R_{2}$. If $a, b \in$ $C=A \times B$, then by definition of characteristic function $\mu_{\chi_{C}}(a)=1=\mu_{\chi_{C}}(b)$ and $\gamma_{\chi_{C}}(a)=0=\gamma_{\chi_{C}}(b)$. Since $a-b$ and $a b \in C, C$ being an LA-subring. It follows that $\mu_{\chi_{C}}(a-b)=1=1 \wedge 1=\mu_{\chi_{C}}(a) \wedge \mu_{\chi_{C}}(b)$ and $\mu_{\chi_{C}}(a b)=$ $1=1 \wedge 1=\mu_{\chi_{C}}(a) \wedge \mu_{\chi_{C}}(b)$. Thus $\mu_{\chi_{C}}(a-b) \geq \min \left\{\mu_{\chi_{C}}(a), \mu_{\chi_{C}}(b)\right\}$ and $\mu_{\chi_{C}}(a b) \geq \min \left\{\mu_{\chi_{C}}(a), \mu_{\chi_{C}}(b)\right\}$. Now $\gamma_{\chi_{C}}(a-b)=0=0 \vee 0=\gamma_{\chi_{C}}(a) \vee$ $\gamma_{\chi_{C}}(b)$ and $\gamma_{\chi_{C}}(a b)=0=0 \vee 0=\gamma_{\chi_{C}}(a) \vee \gamma_{\chi_{C}}(b)$. Thus $\gamma_{\chi_{C}}(a-b) \leq$ $\max \left\{\gamma_{\chi_{C}}(a), \gamma_{\chi_{C}}(b)\right\}$ and $\gamma_{\chi_{C}}(a b) \leq \max \left\{\gamma_{\chi_{C}}(a), \gamma_{\chi_{C}}(b)\right\}$. As $a b$ and $b a \in C$, so $\mu_{\chi_{C}}(a b)=1=\mu_{\chi_{C}}(b a)$ and $\gamma_{\chi_{C}}(a b)=0=\gamma_{\chi_{C}}(b a)$. This imply that $\mu_{\chi_{C}}(a b)=\mu_{\chi_{C}}(b a)$ and $\gamma_{\chi_{C}}(a b)=\gamma_{\chi_{C}}(b a)$. Similarly we have

$$
\begin{aligned}
\mu_{\chi_{C}}(a-b) & \geq \min \left\{\mu_{\chi_{C}}(a), \mu_{\chi_{C}}(b)\right\}, \mu_{\chi_{C}}(a b) \geq \min \left\{\mu_{\chi_{C}}(a), \mu_{\chi_{C}}(b)\right\} \\
\gamma_{\chi_{C}}(a-b) & \leq \max \left\{\gamma_{\chi_{C}}(a), \gamma_{\chi_{C}}(b)\right\}, \gamma_{\chi_{C}}(a b) \leq \max \left\{\gamma_{\chi_{C}}(a), \gamma_{\chi_{C}}(b)\right\} \\
\mu_{\chi_{C}}(a b) & =\mu_{\chi_{C}}(b a) \text { and } \gamma_{\chi_{C}}(a b)=\gamma_{\chi_{C}}(b a)
\end{aligned}
$$

when $a, b \notin C$. Hence the intuitionistic characteristic function $\chi_{C}=$ $\left\langle\mu_{\chi_{C}}, \gamma_{\chi_{C}}\right\rangle$ of $C=A \times B$ is an intuitionistic fuzzy normal LA-subring of $R_{1} \times R_{2}$.

Conversely, assume that the intuitionistic characteristic function $\chi_{C}=$ $\left\langle\mu_{\chi_{C}}, \gamma_{\chi_{C}}\right\rangle$ of $C=A \times B$ is an intuitionistic fuzzy normal LA-subring of $R_{1} \times R_{2}$. Now we have to show that $C=A \times B$ is an LA-subring of $R$. Let 
$a, b \in C$, where $a=\left(a^{\prime}, b^{\prime}\right)$ and $b=\left(a^{\prime \prime}, b^{\prime \prime}\right), a^{\prime}, a^{\prime \prime} \in A, b^{\prime}, b^{\prime \prime} \in B$. By definition $\mu_{\chi_{C}}(a)=1=\mu_{\chi_{C}}(b)$ and $\gamma_{\chi_{C}}(a)=0=\gamma_{\chi_{C}}(b)$. By our supposition

$$
\begin{aligned}
\mu_{\chi_{C}}(a-b) & \geq \mu_{\chi_{C}}(a) \wedge \mu_{\chi_{C}}(b)=1 \wedge 1=1, \\
\mu_{\chi_{C}}(a b) & \geq \mu_{\chi_{C}}(a) \wedge \mu_{\chi_{C}}(b)=1 \wedge 1=1, \\
\gamma_{\chi_{C}}(a-b) & \leq \gamma_{\chi_{C}}(a) \vee \gamma_{\chi_{C}}(b)=0 \vee 0=0, \\
\gamma_{\chi_{C}}(a b) & \leq \gamma_{\chi_{C}}(a) \vee \gamma_{\chi_{C}}(b)=0 \vee 0=0,
\end{aligned}
$$

This imply that $\mu_{\chi_{C}}(a-b)=1, \mu_{\chi_{C}}(a b)=1$ and $\gamma_{\chi_{C}}(a-b)=0, \gamma_{\chi_{C}}(a b)=$ 0 . Thus $a-b$ and $a b \in C$. Hence $C=A \times B$ is an LA-subring of $R_{1} \times R_{2}$.

Theorem 4.4. If $A$ and $B$ are two intuitionistic fuzzy normal LA-subrings of LA-rings $R_{1}$ and $R_{2}$ respectively, then $A \times B$ is an intuitionistic fuzzy normal LA-subring of $R_{1} \times R_{2}$.

Proof. Let $A=\left\{\left(x, \mu_{A}(x), \gamma_{A}(x)\right) \mid x \in R_{1}\right\}$ and $B=\left\{\left(y, \mu_{B}(y), \gamma_{B}(y)\right) \mid y \in\right.$ $\left.R_{2}\right\}$ be intuitionistic fuzzy normal LA-subrings of LA-rings $R_{1}$ and $R_{2}$, respectively. Now $A \times B=\left\{\left((x, y), \mu_{A \times B}((x, y)), \gamma_{A \times B}((x, y))\right) \mid\right.$ for all $x \in R_{1}$ and $\left.y \in R_{2}\right\}$, where $\mu_{A \times B}((x, y))=\min \left\{\mu_{A}(x), \mu_{B}(y)\right\}$ and $\gamma_{A \times B}((x, y))=$ $\max \left\{\gamma_{A}(x), \gamma_{B}(y)\right\}$. We have to show that $A \times B$ is an intuitionistic fuzzy normal LA-subring of $R_{1} \times R_{2}$. Let $(a, b),(c, d) \in R_{1} \times R_{2}$. Now

$$
\begin{aligned}
\mu_{A \times B}((a, b)-(c, d)) & =\mu_{A \times B}((a-c, b-d)) \\
& =\min \left\{\mu_{A}(a-c), \mu_{B}(b-d)\right\} \\
& =\mu_{A}(a-c) \wedge \mu_{B}(b-d) \\
& \geq\left\{\mu_{A}(a) \wedge \mu_{A}(c)\right\} \wedge\left\{\mu_{B}(b) \wedge \mu_{B}(d)\right\} \\
& =\mu_{A}(a) \wedge\left\{\mu_{A}(c) \wedge \mu_{B}(b)\right\} \wedge \mu_{B}(d) \\
& =\mu_{A}(a) \wedge\left\{\mu_{B}(b) \wedge \mu_{A}(c)\right\} \wedge \mu_{B}(d) \\
& =\left\{\mu_{A}(a) \wedge \mu_{B}(b)\right\} \wedge\left\{\mu_{A}(c) \wedge \mu_{B}(d)\right\} \\
& =\mu_{A \times B}((a, b)) \wedge \mu_{A \times B}((c, d)) .
\end{aligned}
$$


and

$$
\begin{aligned}
\mu_{A \times B}((a, b) \circ(c, d)) & =\mu_{A \times B}((a c, b d)) \\
& =\min \left\{\mu_{A}(a c), \mu_{B}(b d)\right\} \\
& =\mu_{A}(a c) \wedge \mu_{B}(b d) \\
& \geq\left\{\mu_{A}(a) \wedge \mu_{A}(c)\right\} \wedge\left\{\mu_{B}(b) \wedge \mu_{B}(d)\right\} \\
& =\mu_{A}(a) \wedge\left\{\mu_{A}(c) \wedge \mu_{B}(b)\right\} \wedge \mu_{B}(d) \\
& =\mu_{A}(a) \wedge\left\{\mu_{B}(b) \wedge \mu_{A}(c)\right\} \wedge \mu_{B}(d) \\
& =\left\{\mu_{A}(a) \wedge \mu_{B}(b)\right\} \wedge\left\{\mu_{A}(c) \wedge \mu_{B}(d)\right\} \\
& =\mu_{A \times B}((a, b)) \wedge \mu_{A \times B}((c, d)) .
\end{aligned}
$$

Thus $A \times B$ is an intuitionistic fuzzy LA-subring. Now

$$
\begin{aligned}
\mu_{A \times B}((a, b) \circ(c, d)) & =\mu_{A \times B}((a c, b d))=\min \left\{\mu_{A}(a c), \mu_{B}(b d)\right\} \\
& =\min \left\{\mu_{A}(c a), \mu_{B}(d b)\right\}, \text { since } A \text { and } B \text { are IFNLSRs } \\
& =\mu_{A \times B}((c a, d b))=\mu_{A \times B}((c, d) \circ(a, b)) .
\end{aligned}
$$

Similarly, $\gamma_{A \times B}((a, b)-(c, d)) \leq \gamma_{A \times B}((a, b)) \vee \gamma_{A \times B}((c, d)), \gamma_{A \times B}((a, b) \circ$ $(c, d)) \leq \gamma_{A \times B}((a, b)) \vee \gamma_{A \times B}((c, d))$ and $\gamma_{A \times B}((a, b) \circ(c, d))=\gamma_{A \times B}((c, d) \circ$ $(a, b))$. Hence $A \times B$ is an intuitionistic fuzzy normal LA-subring of $R_{1} \times$ $R_{2}$.

Theorem 4.5. Let $A$ and $B$ be intuitionistic fuzzy subsets of LA-rings $R_{1}$ and $R_{2}$ with left identity $e_{1}$ and $e_{2}$, respectively and $A \times B$ is an intuitionistic fuzzy LA-subring of $R_{1} \times R_{2}$. Then the following are true.

(1) If $\mu_{A}(x) \leq \mu_{B}\left(e_{2}\right)$ and $\gamma_{A}(x) \geq \gamma_{B}\left(e_{2}\right)$, for all $x \in R_{1}$, then $A$ is an intuitionistic fuzzy LA-subring of $R_{1}$.

(2) If $\mu_{B}(x) \leq \mu_{A}\left(e_{1}\right)$ and $\gamma_{B}(x) \geq \gamma_{A}\left(e_{1}\right)$, for all $x \in R_{2}$, then $B$ is an intuitionistic fuzzy LA-subring of $R_{2}$.

Proof. (1) Let $\mu_{A}(x) \leq \mu_{B}\left(e_{2}\right)$ and $\gamma_{A}(x) \geq \gamma_{B}\left(e_{2}\right)$ for all $x \in R_{1}$, and $y \in R_{1}$. We have to show that $A$ is an intuitionistic fuzzy LA-subring of $R_{1}$. 
Now

$$
\begin{aligned}
\mu_{A}(x-y) & =\mu_{A}(x+(-y))=\min \left\{\mu_{A}(x+(-y)), \mu_{B}\left(e_{2}+\left(-e_{2}\right)\right\}\right. \\
& =\mu_{A \times B}\left(\left(x+(-y), e_{2}+\left(-e_{2}\right)\right)\right. \\
& =\mu_{A \times B}\left(\left(x, e_{2}\right)+\left(-y,-e_{2}\right)\right) \\
& =\mu_{A \times B}\left(\left(x, e_{2}\right)-\left(y, e_{2}\right)\right) \\
& =\mu_{A \times B}\left(\left(x, e_{2}\right)-\left(y, e_{2}\right)\right) \\
& \geq \mu_{A \times B}\left(\left(x, e_{2}\right)\right) \wedge \mu_{A \times B}\left(\left(y, e_{2}\right)\right), \text { since } A \times B \text { is IFLSR } \\
& =\min \left\{\mu_{A}(x), \mu_{B}\left(e_{2}\right)\right\} \wedge \min \left\{\mu_{A}(y), \mu_{B}\left(e_{2}\right)\right\} \\
& =\mu_{A}(x) \wedge \mu_{A}(y) \\
\text { and } \mu_{A}(x y) & =\min \left\{\mu_{A}(x y), \mu_{B}\left(e_{2} e_{2}\right)\right\} \\
& =\mu_{A \times B}\left(\left(x y, e_{2} e_{2}\right)\right) \\
& =\mu_{A \times B}\left(\left(x, e_{2}\right) \circ\left(y, e_{2}\right)\right) \\
& \geq \mu_{A \times B}\left(\left(x, e_{2}\right)\right) \wedge \mu_{A \times B}\left(\left(y, e_{2}\right)\right) \text { since } A \times B \text { is IFLSR } \\
& =\min \left\{\mu_{A}(x), \mu_{B}\left(e_{2}\right)\right\} \wedge \min \left\{\mu_{A}(y), \mu_{B}\left(e_{2}\right)\right\} \\
& =\mu_{A}(x) \wedge \mu_{A}(y)
\end{aligned}
$$

Similarly, we can prove that $\gamma_{A}(x-y) \leq \max \left\{\gamma_{A}(x), \gamma_{A}(y)\right\}$ and $\gamma_{A}(x y) \leq$ $\max \left\{\gamma_{A}(x), \gamma_{A}(y)\right\}$ for all $x, y \in R_{1}$. Thus $A$ is an intuitionistic fuzzy LAsubring of $R_{1}$. (2), is same as (1).

Theorem 4.6. Let $A$ and $B$ be intuitionistic fuzzy subsets of LA-rings $R_{1}$ and $R_{2}$ with left identity $e_{1}$ and $e_{2}$, respectively and $A \times B$ is an intuitionistic fuzzy normal LA-subring of $R_{1} \times R_{2}$. Then the following are true.

(1) If $\mu_{A}(x) \leq \mu_{B}\left(e_{2}\right)$ and $\gamma_{A}(x) \geq \gamma_{B}\left(e_{2}\right)$, for all $x$ in $R_{1}$, then $A$ is an intuitionistic fuzzy normal LA-subring of $R_{1}$.

(2) If $\mu_{B}(x) \leq \mu_{A}\left(e_{1}\right)$ and $\gamma_{B}(x) \geq \gamma_{A}\left(e_{1}\right)$, for all $x$ in $R_{2}$, then $B$ is an intuitionistic fuzzy normal LA-subring of $R_{2}$.

Proof. Let $A \times B$ be an intuitionistic fuzzy normal LA-subring of $R_{1} \times R_{2}$.

(1) Let $\mu_{A}(x) \leq \mu_{B}\left(e_{2}\right)$ and $\gamma_{A}(x) \geq \gamma_{B}\left(e_{2}\right)$ for all $x$ in $R_{1}$, and let $y \in R_{1}$. Now we have to show that $A$ is an intuitionistic fuzzy normal LA-subring of $R_{1}$. Since $A$ is an intuitionistic fuzzy LA-subring of $R_{1}$, by Theorem 4 (1). 
Now

$$
\begin{aligned}
\mu_{A}(x y) & =\min \left\{\mu_{A}(x y), \mu_{B}\left(e_{2} e_{2}\right)\right\} \\
& =\mu_{A \times B}\left(\left(x y, e_{2} e_{2}\right)\right) \\
& =\mu_{A \times B}\left(\left(x, e_{2}\right) \circ\left(y, e_{2}\right)\right) \\
& =\mu_{A \times B}\left(\left(y, e_{2}\right) \circ\left(x, e_{2}\right)\right), \text { since } A \times B \text { is an IFNLSR } \\
& =\mu_{A \times B}\left(\left(y x, e_{2} e_{2}\right)\right) \\
& =\min \left\{\mu_{A}(y x), \mu_{B}\left(e_{2} e_{2}\right)\right\} \\
& =\mu_{A}(y x)
\end{aligned}
$$

Similarly $\gamma_{A}(x y)=\gamma_{A}(y x)$. Hence $A$ is an intuitionistic fuzzy normal LA-subring of $R_{1}$.

(2) Let $\mu_{B}(x) \leq \mu_{A}\left(e_{1}\right)$ and $\gamma_{B}(x) \geq \gamma_{A}\left(e_{1}\right)$ for all $x$ in $R_{2}$, and let $y \in R_{2}$. Now we have to show that $B$ is an intuitionistic fuzzy normal LA-subring of $R_{2}$. Since $B$ is an intuitionistic fuzzy LA-subring of $R_{2}$, by Theorem 4 (2). Now

$$
\begin{aligned}
\mu_{B}(x y) & =\min \left\{\mu_{A}\left(e_{1} e_{1}\right), \mu_{B}(x y)\right\} \\
& =\mu_{A \times B}\left(\left(e_{1} e_{1}, x y\right)\right) \\
& =\mu_{A \times B}\left(\left(e_{1}, x\right) \circ\left(e_{1}, y\right)\right) \\
& =\mu_{A \times B}\left(\left(e_{1}, y\right) \circ\left(e_{1}, x\right)\right), \text { since } A \times B \text { is an IFNLSR } \\
& =\mu_{A \times B}\left(\left(e_{1} e_{1}, y x\right)\right) \\
& =\min \left\{\mu_{A}\left(e_{1} e_{1}\right), \mu_{B}(y x)\right\} \\
& =\mu_{B}(y x) .
\end{aligned}
$$

Similarly, $\gamma_{B}(x y)=\gamma_{B}(y x)$. Hence $B$ is an intuitionistic fuzzy normal LA-subring of $R_{2}$.

\section{Conclusion}

Though the study of fuzzy sets, where the base crisp set is a commutative ring, has attracted the attention of many researchers over many years. Even then, many sets are naturally endowed with two compatible operations forming a non-commutative and non-associative ring. In this context, we can find examples showing that the fuzzy properties must not be restricted for commutative rings. Thus it seems natural to study fuzzy sets over non-commutative and non-associative rings. In this paper, we initiated the concept of intuitionistic fuzzy normal LA-subrings of LA-rings. We extended the notion of intuitionistic fuzzy normal subrings to a non-associative class of LA-rings. Also we established the direct product of LA-rings and derived some related properties. Though LA-ring is a non-associative and non-commutative structure, 
but due to its peculiar characteristics, it possesses properties which we usually encounter in associative algebraic structures. In future we hope that, this concept would have a useful contribution in the application of non-associative algebraic structures.

\section{References}

[1] K. Atanassov, Intuitionistic fuzzy sets, Fuzzy Sets and Systems 20 (1986), 87-96.

[2] K. Atanassov, New operations defined over the intuitionistic fuzzy sets, Fuzzy Sets and systems 61 (1994), 137-142.

[3] B. Banerjee and D. K. Basnet, Intuitionistic fuzzy subrings and ideals, J. Fuzzy Math. 11 (2003), 139-155.

[4] R. Biswas, Intuitionistic fuzzy subrings, Mathematical Forum x (1989), $37-46$.

[5] R. J. Cho, J. Jezek and T. Kepka praha, Paramedial groupoids, Czechoslovak Mathematical Journal 49 (1999), 391-399.

[6] P. Corsini ${ }^{1}$ and I. Cristea ${ }^{2}$, Fuzzy sets and non complete 1-Hypergroups ${ }^{*}$, An. St. Univ. Ovidius Constant 13 (2005), 27-54.

[7] I. Cristea, Complete Hypergroups, 1-Hypergroups and fuzzy sets, An. St. Univ. Ovidius Constant 10 (2002), 25-38.

[8] K. A. Dib and N. L. Youssef, Fuzzy Cartesian product, fuzzy relations and fuzzy functions, Fuzzy Sets Syst 41 (1991), 299-315.

[9] K. A. Dib, N. Galhum and A. A. M. Hassan, Fuzzy rings and fuzzy ideals, Fuzzy Math. 4 (1996), 245-261.

[10] D. Dubois, S. Gottwaldb, P. Hajekc, J. Kacprzykd and H. Pradea, Terminological difficulties in fuzzy set theory-The case of "Intuitionistic Fuzzy Sets", Fuzzy Sets and Systems 156 (2005), 485-491.

[11] P. Holgate, Groupoids satisfying a simple invertive law, Math. Stud. 61 (1992), 101-106.

[12] K. Hur*, H. w. Kang and H. k. Song, Intuitionistic Fuzzy subgroups and subrings, Honam Math. J. 25 (2003), 19-41.

[13] J. Jezek, T. Kepka, Medial groupoids, Rozpravy CSAV 93/2(1983). 
[14] M. A. Kazim, M. Naseerudin, On almost semigroups, Alig. Bull. Math. 2 (1972), 1-7.

[15] M. S. Kamran, Conditions for LA-semigroups to resemble associative structures, Ph.D. Thesis, Quaid-i-Azam University, Islamabad, 1993.

[16] M. Khan and M. N. A. Khan, On Fuzzy Abel Grassmann's Groupoids, AFM 5 (2010), 349-360.

[17] S. Narmada and V. M. Kumar, Intuitionistic Fuzzy Bi-Ideals and Regularity in Near-Rings, International Journal of Algebra 5 (2011), 483-490.

[18] M. F. Marashdeh and A. R. Salleh, Intuitionistic Fuzzy Rings, Int. J. Algebra 5 (2011), 37-47.

[19] N. Palaniappan and K. Arjunan, Some properties of intuitionistic fuzzy subgroups, Acta Ciencia Indic Math. 2 (2007), 321-328.

[20] N. Palaniappan, K. Arjunan and V. Veeramani, The homomorphism, antihomomorphism of an intuitionistic fuzzy normal subrings, Acta Ciencia Indica Math. 219 (2007), 219-224.

[21] P. V. Protic and N. Stevanovic, AG-test and some general properties of Abel-Grassmann's groupoids, Pure Math. and applications 6 (1995), 371383.

[22] P. V. Protic and N. Stevanovic, The structural theorem for $A G^{*}$ groupoids, Series Mathematics Informatics 10 (1995), 25-33.

[23] T. Shah and N. Kausar, Fuzzy ideals in LA-rings (submitted).

[24] T. Shah and N. Kausar, Intuitionistic fuzzy bi-ideals in LA-rings (submitted).

[25] T. Shah and I. Rehman, On LA-rings of finitely non-zero functions, Int. J. Contemp. Math. Sciences 5 (2010), 209-222.

[26] T. Shah and I. Rehman, On characterizations of LA-rings through some properties of their ideals, Southeast Asian Bull. Math. (to appear).

[27] M. Shah and T. Shah, Some basic properties of LA-rings, Int. Math. Forum 6 (2011), 2195-2199.

[28] V. Veeramani, K. Arjunan and N. Palaniappan, Some Properties of Intuitionistic Fuzzy Normal Subrings, Applied Math. Sciences 4 (2010), 2119-2124. 
[29] A. C. Volf, Fuzzy subfields, An. S,t. Univ. Ovidius Constant,a vol. 9, no. 2(2001), 193-198.

[30] L. M. Yan, Intuitionistic Fuzzy Ring and Its Homomorphism Image, Int. Seminar on Future BioMedical Infor. Eng. fbie, (2008), 75-77.

[31] L. A. Zadeh, Fuzzy sets, Information and Control 8 (1965), 338-353.

Tariq Shah,

Department of Mathematics,

Quaid-i-Azam University Islamabad-Pakistan,

Email: stariq@gmail.com

Nasreen Kausar,

Department of Mathematics,

Quaid-i-Azam University Islamabad-Pakistan,

Email: kausar.nasreen57@gmail.com

Inayatur-Rehman,

Department of Mathematics,

COMSATS, Institute of Information Technology, Abbottabad-Pakistan,

Email:sinayat@ciit.net.pk 